\title{
A Systematic Review and Meta-Analysis of Treatment and Outcome of Blister-Like Aneurysms
}

\author{
(D). Peschillo, (DD. Cannizzaro, (D)A. Caporlingua, and DP. Missori
}

\begin{abstract}
BACKGROUND AND PURPOSE: Blister-like aneurysms are uncommon but challenging lesions. Their small size and atypical location often make the diagnosis difficult. Microsurgery and endovascular procedures have been used for their treatment, but there is no consensus on the best treatment technique. We conducted a systematic review and meta-analysis of treatments and outcomes of these vascular lesions.
\end{abstract}

MATERIALS AND METHODS: We reviewed English-language articles on "blood blister-like aneurysms" published between January 1997 and November 2014. All studies reporting patients with these aneurysms treated with surgery, endovascular procedures, or combined therapy with data on treatment modalities and clinical and/or angiographic outcomes were selected, including case reports and series. We performed a meta-analysis on the 2 largest treatment groups (surgery and endovascular management).

RESULTS: Sixty studies with 334 patients met our inclusion criteria. Surgery was performed in 114 patients (34.2\%), and endovascular treatment, in 199 patients (59.5\%). A combined treatment was used in 19 patients (5.7\%). A favorable outcome (mRS $0-2$ ) was reported in $67.4 \%$ and $78.9 \%$ of patients treated with surgery and with endovascular therapy, respectively $(P=.034)$.

CONCLUSIONS: Blister-like aneurysms are challenging vascular lesions. The choice of treatment method must be based on the initial clinical presentation and an analysis of the radiologic features of the lesion to select the best technique. Endovascular treatment seems to have lower morbidity and mortality and provides a better outcome compared with surgical approaches. Further prospective studies must be performed to confirm such interesting results.

ABBREVIATION: $\mathrm{HH}=$ Hunt and Hess

B lister-like aneurysms, described for the first time by Nakagawa et $\mathrm{al}^{1}$ and Takahashi et $\mathrm{al},{ }^{2}$ account for approximately $0.3 \%-1 \%$ of all intracranial aneurysms ${ }^{3}$ and are a therapeutic challenge. They typically have thin, fragile walls and poorly defined necks, sometimes appearing as just a protrusion of the vessel wall. They are usually associated with diffuse subarachnoid hemorrhage and a severe clinical condition. These lesions can be dangerous because of their propensity to rupture intraoperatively and

Received July 14, 2015; accepted after revision September 8.

From the Department of Neurology and Psychiatry, Endovascular Neurosurgery/ Interventional Neuroradiology (S.P.); and Neurosurgery (D.C., A.C., P.M.), "Sapienza," University of Rome, Rome, Italy.

S.P. had the conception and design of the work; all authors contributed equally to drafting and revising the manuscript and all gave final approval of the version published. D.C. selected the studies for inclusion.

Please address correspondence to Simone Peschillo, MD, PhD, Department of Neurology and Psychiatry, Endovascular Neurosurgery/Interventional Neuroradiology, "Sapienza" University of Rome, Rome, Italy; e-mail: simone.peschillo@gmail.com

- Indicates open access to non-subscribers at www.ajnr.org

http://dx.doi.org/10.3174/ajnr.A4606 postoperatively. Blister-like aneurysms are often not easily detected at the first angiography due to their small dimensions and unusual locations, but because of their rapid growth, they become more evident within a few days.

Originally, they were thought to occur exclusively in the internal carotid artery; however, recent studies have shown that this type of vascular lesion can be found anywhere in the circle of Willis. Specifically, there have been reports of blisterlike aneurysms in the middle cerebral artery, anterior communicating artery, and vertebrobasilar circulation. ${ }^{4-7}$

The above characteristics make them particularly difficult to treat, and patients with these lesions often have a poor outcome. Numerous treatment methods have been proposed: microsurgery, including clipping techniques, titanium vascular miniclip internal carotid artery repair, vessel graft wrapping reinforced by clips, trapping, parent vessel occlusion, and revascularization; and endovascular approaches, which can be performed with coils, coils and stent placement, multiple stents, stent placement alone, or vessel occlusion. Flow-diverting stents have been used to treat these lesions with good early 
results, but their long-term safety has yet to be proved. The current literature describing surgical and endovascular interventions in blister-like aneurysms does not offer a clear consensus on the optimal treatment technique. ${ }^{8}$

The aim of this work was to perform a systematic review and meta-analysis of the various types of treatment to compare their efficacy and safety.

\section{MATERIALS AND METHODS}

A comprehensive literature search of the PubMed and Scopus data bases was conducted by using the key words "blood blisterlike aneurysm," "blister-like aneurysm," and "blister aneurysm." The search was limited to articles published between January 1997 and November 2014 in English only. All studies reporting patients with blister-like aneurysms treated with surgery, endovascular procedures, or combined therapy were selected. Evaluation was performed according to the "Preferred Reporting Items for Systematic Reviews and Meta-Analyses" (PRISMA) statement, including methods of publication search, eligibility, data collection, extraction, analysis, and preparation of the systematic review report.

Case reports or series with available data on clinical and/or angiographic outcomes and details of the treatment modalities were included. One reviewer (D.C.) selected the studies for inclusion.

For each study, we extracted the following information: patient presentation described by using validated scales (Hunt and Hess $[\mathrm{HH}]$ and Fisher), treatment technique (endovascular, surgical, combined), and long-term neurologic outcome (a good neurologic outcome was defined as a modified Rankin Scale score of $\leq 2$ ). When an mRS score was not available, good neurologic outcome was determined from the description of the clinical results (eg, terms such as "no morbidity" or "good recovery").

For the analysis, we have defined angiographic occlusion status immediately after treatment and at the last follow-up as "complete," "near-complete," or "incomplete."

The primary objective of this study was to determine the clinical and angiographic outcomes of patients treated with endovascular procedures or surgery (the group of patients that underwent combined treatment was excluded from the statistical analysis because of its small size $[n=19])$. The secondary objective was to determine perioperative, intraoperative, and postoperative complications in the endovascular and surgical groups, morbidity at the last follow-up, and the clinical outcome in relation to the technique used.

\section{Literature Review}

Our literature search initially yielded 103 articles; 43 articles were excluded either because the patients did not have blister-like aneurysms or because the patients' presentations or angiographic outcomes were not described. In total, 60 studies with 334 patients met our inclusion criteria.

\section{Statistical Analysis}

Statistical analyses were performed by using SPSS, Release 15.0 (IBM, Armonk, New York). All included studies were non-
Table 1: Distribution of locations of blister-like aneurysms

\begin{tabular}{lc}
\multicolumn{1}{c}{ Topography } & (\%) \\
\hline Internal carotid artery & 91.6 \\
Anterior communicating artery & 2.8 \\
Middle cerebral artery & 1.9 \\
Posterior cerebral artery & 1.6 \\
Basilar artery & 1.6 \\
Anterior cerebral artery & 0.3 \\
Total & 100 \\
\hline
\end{tabular}

comparative. From each cohort, we estimated the cumulative incidence (event rate) and 95\% confidence interval for each outcome. Event rates for each intervention were pooled in a meta-analysis across studies. Frequencies of categoric variables of pooled data were compared by using the $\chi^{2}$ test. Finally, a multivariable binary logistic analysis was performed to evaluate variables predictive of clinical outcome. Variables included in the model were age, sex, treatment (surgery/endovascular), and Fisher and $\mathrm{HH}$ grades. The coefficients obtained from the logistic regression were expressed in terms of odds ratios with $95 \%$ confidence intervals. All tests were 2 -sided, and statistical significance was set at $P<.05$.

\section{RESULTS}

We selected 60 studies, including retrospectively collected case series and case reports, evaluating 334 patients. A preponderance of females was noted: There were 246 females (73\%) and 88 males. The average age of the patients in these studies was 48.5 years (range, $14-87$ years). The median $\mathrm{HH}$ grade at admission was $3 ; 59$ patients had an $\mathrm{HH}$ grade of $\geq 4$ on admission (44 patients had grade $4 ; 15$ had grade 5). More than $90 \%$ of the blister-like aneurysms were located along nonbranching sites of the internal carotid artery (91.6\%); all of those arising from intracranial arteries other than the internal carotid artery were classified as "atypical." The sites are presented in Table 1. A treatment was offered to nearly all patients evaluated; only 2 patients were managed conservatively $(0.6 \%)$. Surgery was performed in 114 patients (34.2\%); an endovascular approach was preferred in 199 cases (59.5\%), while a combined approach (either a pure combined approach or salvage therapy following failure of the initial therapeutic technique) was used in 19 patients $(5.7 \%)$. For statistical purposes, we focused on the 2 more frequently used types of treatment (surgery and endovascular procedures).

As mentioned before, the primary objective of this study was to determine the clinical and angiographic outcomes of patients treated with endovascular approaches or surgery. Regarding outcome, based on a division of the mRS scores into 2 groups $(0,1$, or 2 and $3,4,5$, or 6 ), $70 \%$ of patients with $\mathrm{HH}$ grade $\leq 3$ in the group treated with surgery had a positive outcome (mRS $0-2$ ), compared with $84.9 \%$ of the patients in the endovascularly treated group $(P=.008)$. Among the patients with $\mathrm{HH}$ grade $\geq 4$, only $59.1 \%$ of the surgical group had a positive outcome (mRS $0-2$ ), and the percentage in the endovascularly treated group was even lower at $40.7 \%(P=.251)$ (Table 2$)$.

Considered together, including patients with all $\mathrm{HH}$ grades, $67.4 \%$ of the group treated with surgery had an mRS score of 0,1 , or 2 , while $32.6 \%$ had an mRS score of $3,4,5$, or 6 . The corre- 
sponding percentages in the endovascular group were $78.9 \%$ and 21.1\% $(P=.034)$ (Table 2).

When we analyzed the outcome of patients divided on the basis of the Fisher scale and treatment received, among the pa-

Table 2: Clinical outcomes according to type of treatment and presentation

\begin{tabular}{ccccccc}
\hline & $\begin{array}{c}\text { Surgery-Only } \\
\text { Group Outcome } \\
\text { (mRS Score) }\end{array}$ & & \multicolumn{2}{c}{$\begin{array}{c}\text { Endovascular } \\
\text { Group Outcome } \\
\text { (mRS Score) }\end{array}$} & \\
\cline { 2 - 3 } & $\mathbf{0 , 1 , 2}$ & $\mathbf{3 , 4 , 5 , 6}$ & & $\mathbf{0 , 1 , 2}$ & $\mathbf{3 , 4 , 5 , 6}$ & \\
\hline Hunt Hess & & & & & \\
$\quad$ All subjects & $67.4 \%$ & $32.6 \%$ & & $78.9 \%$ & $21.1 \%$ & $.034^{\mathrm{a}}$ \\
$\mathrm{HH} \leq 3$ & $70.0 \%$ & $30.0 \%$ & & $84.9 \%$ & $15.1 \%$ & $.006^{\mathrm{a}}$ \\
$\mathrm{HH} \geq 4$ & $59.1 \%$ & $40.9 \%$ & & $40.7 \%$ & $59.3 \%$ & .251 \\
Fisher & & & & & \\
All subjects & $67 \%$ & $33 \%$ & & $77.8 \%$ & $22.2 \%$ & $.054^{\mathrm{a}}$ \\
Fisher 1-2 & $77.1 \%$ & $22.9 \%$ & & $88.8 \%$ & $11.2 \%$ & .081 \\
Fisher 3-4 & $61.4 \%$ & $38.6 \%$ & $61.1 \%$ & $38.9 \%$ & .973 \\
\hline
\end{tabular}

a Significant.

Table 3: Multivariable binary logistic analysis to evaluate variables predictive of clinical outcome

\begin{tabular}{lccc}
\hline \multicolumn{1}{c}{ Variable } & $\boldsymbol{\beta} \pm \mathbf{S E}$ & $\boldsymbol{P}$ Value & OR $(95 \% \mathrm{CI})$ \\
\hline Treatment (surgery/ & $-0.20 \pm 0,31$ & .516 & $0.82(0.45-1.49)$ \\
$\quad$ endovascular) & & & \\
Sex (female/male) & $-0.07 \pm 0.35$ & .840 & $1.02(0.99-1.05)$ \\
Age (yr) & $0.02 \pm 0.01$ & .197 & $0.93(0.47-1.85)$ \\
Fisher grade & $0.44 \pm 0.20$ & $.031^{\mathrm{a}}$ & $1.55(1.04-2.30)$ \\
HH grade & $0.38 \pm 0.19$ & $.041^{\mathrm{a}}$ & $1.47(1.02-2.12)$ \\
\hline
\end{tabular}

Note:-SE indicates standard error.

a Significant. tients with Fisher grade $\leq 2$, the outcome was good (mRS 0-2) in $77.1 \%$ of patients in the surgery group and in $88.8 \%$ of those in the endovascular group $(P=.081)$. In contrast, among the patients with Fisher grade $\geq 3$, the percentages of patients with a good outcome were essentially the same $(61.4 \%$ in the surgery group versus $61.1 \%$ in the endovascular group; $P=.973)$. In multivariable analysis (Table 3), both the $\mathrm{HH}$ and Fisher grade were clear predictors of outcome ( $P=.041$ and $P=.031$, respectively).

Concerning angiographic outcome, the frequencies of early occlusion status (complete, near-complete, and incomplete) were $96.4 \%, 3.6 \%$, and $0.0 \%$, respectively, in the surgery group and $44.5 \%, 41.0 \%$, and $14.5 \%$, respectively, in the endovascular group $(P<.001)$. The frequencies of follow-up occlusion status were $97.3 \%, 2.7 \%$, and $0.0 \%$, respectively, in the surgery group and $76.9 \%, 18.1 \%$, and $5.0 \%$, respectively, in the endovascular group $(P<.001)$.

Regarding morbidity and mortality, perioperative morbidity occurred in $7.0 \%$ (95\% CI, 3.0\%-19.0\%) of the endovascular group and $20.0 \%(95 \% \mathrm{CI}, 6.0 \%-49.0 \%)$ of the surgery group. Perioperative death occurred in $9.0 \%$ (95\% CI, 5.0\%-13.0\%) of the endovascular group and $10.7 \%$ (95\% CI, 5.0\%-16.4\%) of the surgery group. However, when the population was split into 2 subgroups according to $\mathrm{HH}$ grade, among subjects with an $\mathrm{HH}$ grade of $<4$, the mortality rate was significantly higher in the surgery group than in the endovascular group (14.3\% versus $4.6 \%$, respectively; $P=.009$ ); on the other hand, for patients with an $\mathrm{HH}$ grade of $>3$, the mortality was higher in the endovascular group $(37.0 \%$ versus $9.1 \%, P=.043)$.

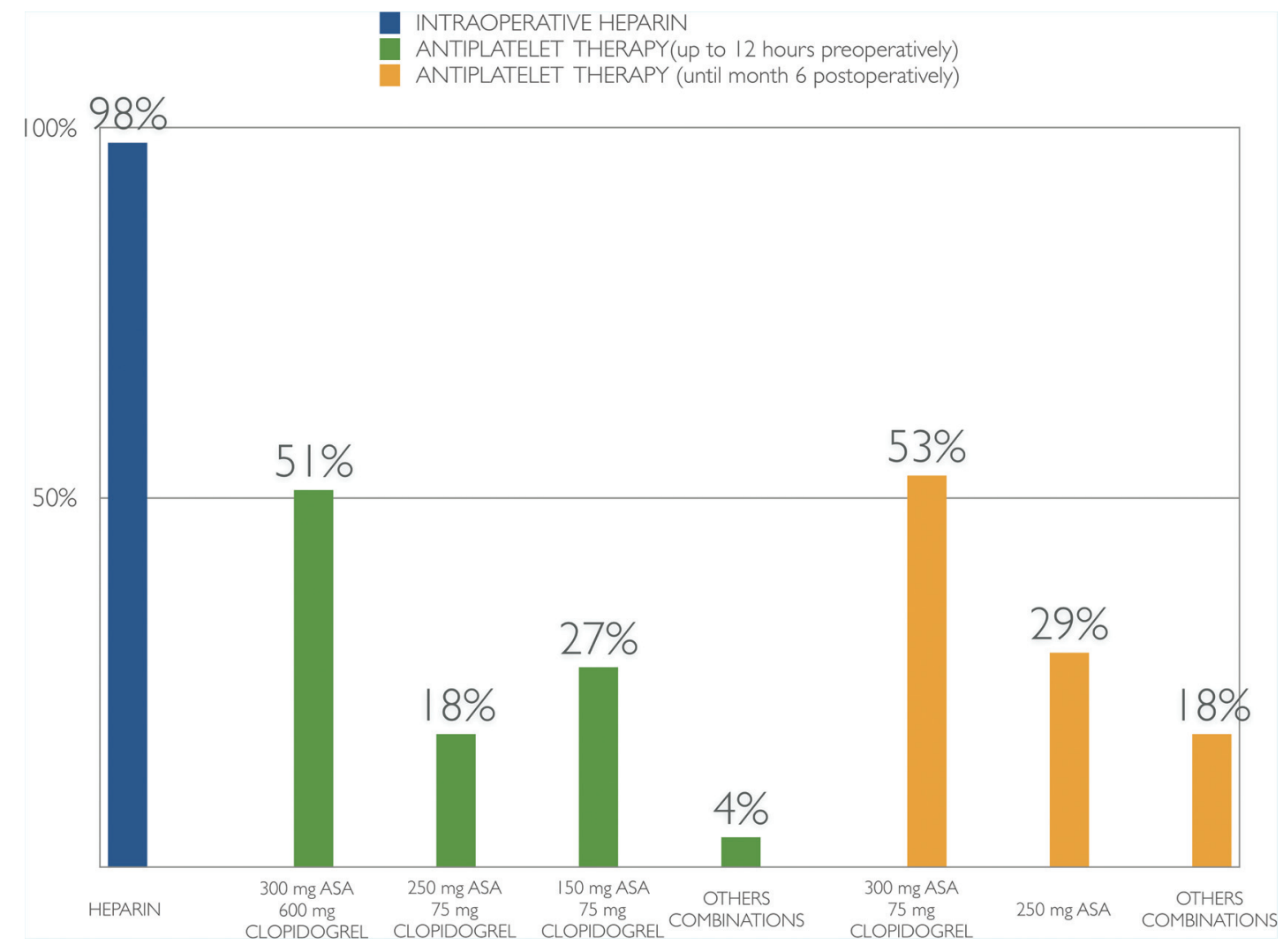

FIG 1. The graph shows the different data concerning antiplatelet therapy administered to patients treated with flow-diverter stents intraoperatively, up to 12 hours before the procedure, and during the first 6 postoperative months. Note that in some cases, this information was missing. These statistics are based on the available data. ASA indicates aspirin. 


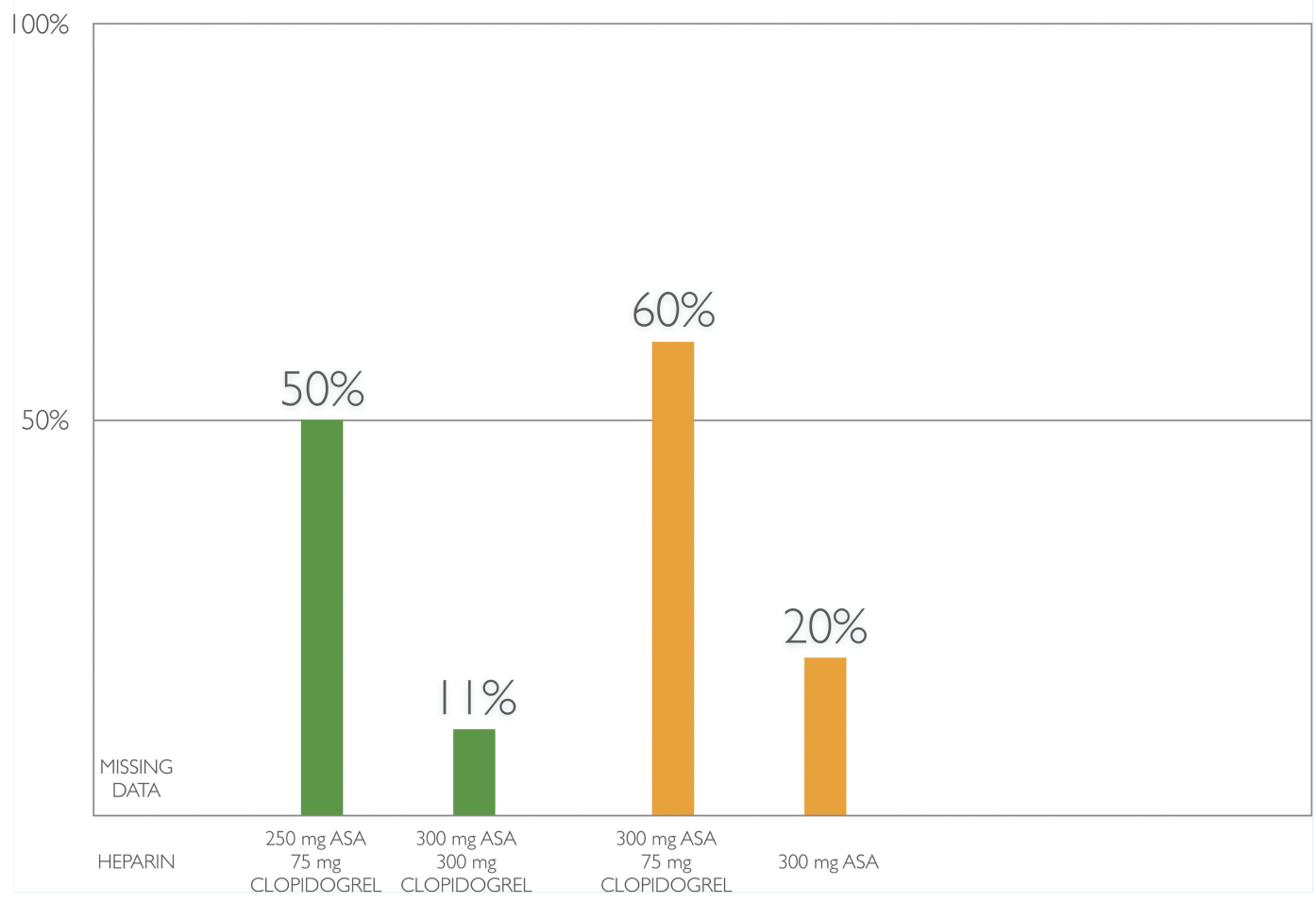

FIG 2. The graph shows the different data concerning antiplatelet therapy administered to patients treated with no flow-diverter stent up to 12 hours before the procedure, and during the first 6 postoperative months. Note that in some cases this information was missing. These statistics are based on the available data.

Intraprocedural complications occurred more frequently in the surgery group $(24.1 \%)$ than in the endovascular group $(10.5 \%)(P<.001)$. The most frequent complication in the surgery group was rupture (81.5\%). In the endovascular group, the most feared complications were thromboembolic events (33.3\%), followed by vasospasm (19\%); in particular, thromboembolic events were much more frequent in flow-diverter procedures (50\%).

Concerning antiplatelet treatment in the endovascular group, it is worth considering the experiences of various authors when flow-diverter stents were or were not used (Figs 1 and 2).

Postprocedural complications were reported in $35.7 \%$ of the surgery group and $21.1 \%$ of the endovascular group $(P<.001)$. The most frequent complications in the surgery group were hydrocephalus and stroke in $20.0 \%$ and vasospasm in $17.5 \%$, while the most common complications in the endovascular group were recanalization and hemorrhage, which occurred in $54.8 \%$ and $14.3 \%$, respectively.

The outcomes in the endovascular group differed according to the endovascular technique used, with the highest percentages of patients having a good outcome being among those treated with stents $(86.4 \%)$, stent-assisted coiling $(85.2 \%)$, and flow diverters $(82.2 \%)$; the subgroup with the lowest rate of good outcomes (mRS $0-2)$ was treated with coils $(52.9 \%)(P=$ .266) (Table 4). In the surgery group, the highest rates of good outcomes were found in the subgroups managed with trapping + radial artery bypass $(92.3 \%)$, wrapping + clipping $(82.9 \%)$, and clipping + arteriotomy $(68.4 \%)(P=.12)$ (Table 5). These dif-
Table 4: Clinical outcome of patients treated with endovascular techniques

\begin{tabular}{|c|c|c|c|c|c|c|}
\hline & \multicolumn{4}{|c|}{$\begin{array}{c}\text { Outcome (mRS Score) } \\
\qquad(P=.266)\end{array}$} & \multirow[b]{3}{*}{ Total } & \multirow[b]{3}{*}{ (\%) } \\
\hline & \multicolumn{2}{|c|}{$0,1,2$} & \multicolumn{2}{|c|}{$3,4,5,6$} & & \\
\hline & (No.) & (\%) & (No.) & $(\%)$ & & \\
\hline Balloon-assisted coils & 6 & 75.0 & 2 & 25.0 & 8 & 4 \\
\hline Coil & 9 & 52.9 & 8 & 47.1 & 17 & 9 \\
\hline Coil + stent, no FD & 41 & 71.9 & 16 & 28.1 & 57 & 29 \\
\hline FD & 37 & $82.2^{\mathrm{a}}$ & 8 & $17.8^{\mathrm{a}}$ & 45 & 23 \\
\hline FD + coils & 3 & 100.0 & 0 & 0.0 & 3 & 2 \\
\hline FD + stent & 1 & 100.0 & 0 & 0.0 & 1 & 1 \\
\hline Multistent & 2 & 100.0 & 0 & 0.0 & 2 & 1 \\
\hline PAO & 8 & 88.9 & 1 & 11.1 & 9 & 5 \\
\hline SAC & 23 & $85.2^{\mathrm{a}}$ & 4 & $14.8^{a}$ & 27 & 14 \\
\hline$S A C+F D$ & 1 & 100.0 & 0 & 0.0 & 1 & 1 \\
\hline Stent & 19 & $86.4^{\mathrm{a}}$ & 3 & $13.6^{\mathrm{a}}$ & 22 & 11 \\
\hline Stent in stent & 3 & 100.0 & 0 & 0.0 & 3 & 2 \\
\hline Trapping with coil & 4 & 100.0 & 0 & 0.0 & 4 & 2 \\
\hline Total & 157 & & 42 & & 199 & 104 \\
\hline
\end{tabular}

Note:-FD indicates flow diverter; PAO, parent artery occlusion; SAC, stent-assisted coiling,

${ }^{\text {a }}$ Results for the largest groups.

ferences were not statistically significant and should, therefore, be interpreted with caution.

\section{DISCUSSION}

Blister-like aneurysms were first described by Nakagawa et $\mathrm{al}^{1}$ and Takahashi et al. ${ }^{2}$ The pathogenesis of these vascular disorders remains unclear, but several mechanisms have been proposed, in- 
Table 5: Clinical outcome of patients treated with surgical techniques

\begin{tabular}{|c|c|c|c|c|c|c|}
\hline & \multicolumn{4}{|c|}{$\begin{array}{c}\text { Outcome (mRS Score) } \\
(P=.12)\end{array}$} & \multirow[b]{3}{*}{ Total } & \multirow[b]{3}{*}{ (\%) } \\
\hline & \multicolumn{2}{|c|}{$0,1,2$} & \multicolumn{2}{|c|}{$3,4,5,6$} & & \\
\hline & (No.) & $(\%)$ & (No.) & $(\%)$ & & \\
\hline Bypass & 1 & 100.0 & 0 & 0.0 & 1 & 0.9 \\
\hline Clip & 4 & 66.7 & 2 & 33.3 & 6 & 5.3 \\
\hline Clip + arteriotomy & 26 & $68.4^{\mathrm{a}}$ & 12 & $31.6^{\mathrm{a}}$ & 38 & 33.6 \\
\hline Clip + bypass & 3 & 100.0 & 0 & 0.0 & 3 & 2.7 \\
\hline PAO & 0 & 0.0 & 1 & 100.0 & 1 & 0.9 \\
\hline $\begin{array}{l}\text { Proximal ligation }+ \text { RA } \\
\text { bypass }\end{array}$ & 0 & 0.0 & 1 & 100.0 & 1 & 0.9 \\
\hline Sutured & 2 & 66.7 & 1 & 33.3 & 3 & 2.7 \\
\hline Trapping & 2 & 22.2 & 7 & 77.8 & 9 & 8.0 \\
\hline Trapping + RA bypass & 12 & $92.3^{a}$ & 1 & $7.7^{\mathrm{a}}$ & 13 & 11.5 \\
\hline $\begin{array}{l}\text { Wrapping + bypass }+ \\
\text { PAO }\end{array}$ & 1 & 100.0 & 0 & 0.0 & 1 & 0.9 \\
\hline Wrapping + clip & 29 & $82.9^{\mathrm{a}}$ & 6 & $17.1^{\mathrm{a}}$ & 35 & 31.0 \\
\hline Wrapping & 1 & 50.0 & 1 & 50.0 & 2 & 1.8 \\
\hline Total & 81 & & 32 & & 113 & 100 \\
\hline
\end{tabular}

Note:-RA indicates radial artery; PAO, parent artery occlusion.

${ }^{a}$ Results for the largest groups.

Table 6: Characteristics of blister-like aneurysms

\begin{tabular}{ll}
\multicolumn{1}{c}{ Characteristic } & \multicolumn{1}{c}{ Description } \\
\hline Localization & Nonbranching site, proximal \\
Dimension & Small, $<3$ mm (within 24 hours of SAH) \\
Shape & Conical, wide-neck \\
Appearance & Dissecting \\
Tendency to rebleed & High, due to friable walls \\
Tendency to grow & High \\
\hline
\end{tabular}

cluding atherosclerosis, hemodynamic stress related to hypertension, and arterial dissection creating a pseudoaneurysm. A postmortem pathologic study performed by Ishikawa et $\mathrm{al}^{3}$ demonstrated blister-like aneurysms that arose in an atherosclerotic carotid artery in the junction between the sclerotic and normal carotid wall; there was degeneration of the internal elastic lamina associated with a lack of normal adventitia and fibrinous tissue at this site, leading to laceration of the internal carotid wall, suggesting that these kinds of vascular lesions may be a subtype of dissecting aneurysms or pseudoaneurysms. ${ }^{3,9}$

Although a definitive diagnosis can only be made from histologic studies, numerous features define blister-like aneurysms. According to the literature, we can define these as a small aneurysm ( $<3 \mathrm{~mm}$, within 24 hours from subarachnoid hemorrhage), located at a nonbranching site, and proximal to the circle of Willis, which can evolve during a brief period. This type of aneurysm has friable walls, making the risk of intraoperative and postoperative bleeding high. It is conical and wide-necked with a dissecting/nonsaccular appearance (Table 6). It is frequently unrecognized at initial digital subtraction angiography.

Numerous treatments have been proposed, including microsurgical and endovascular techniques, without a unanimous consensus on the best method having been reached, probably because of the rarity of these lesions, which account for $<1 \%$ of all intracranial aneurysms.

The aim of this meta-analysis was an overall analysis of this type of lesion and the various treatment options to gain a better understanding of the preferable therapeutic choices.
As reported in the "Results," endovascular treatment was associated with a higher rate of favorable outcomes if the patients presented with an $\mathrm{HH}$ grade of $\leq 3$; however, in more severe cases $(\mathrm{HH} \geq 4)$, the outcome was better in the group treated with surgery. Overall, including patients with all $\mathrm{HH}$ grades, $67.4 \%$ in the group treated surgically had a good outcome (mRS 0-2), while $32.6 \%$ had a less satisfactory outcome (mRS 3-6) (Table 2). The corresponding percentages in the group treated with endovascular approaches were $78.9 \%$ and $21.1 \%(P=.034)$ (Table 2). The Fisher grade is also a good predictor of outcome; in fact, of the patients with Fisher grade $\leq 2,77.1 \%$ in the surgery group had a positive outcome (mRS $0-2$ ) as did $88.8 \%$ in the endovascular group $(P=.081)$. Both $\mathrm{HH}$ and Fisher grades remained clear predictors of outcome in the multivariable analysis $(P=.041$ and $P=.031$, respectively) (Table 3 ).

Concerning angiographic outcome, surgery was more likely to achieve early, complete occlusions, whereas there was an improvement of $32.4 \%$ between the early occlusion status and the follow-up occlusion status in the endovascular group. This difference did not, however, seem to influence the outcome; it appears clear that the clinical status at the outset has a much greater effect than complete occlusion of the aneurysm.

Intraprocedural complications were more frequent in the surgery group than in the endovascular one $(24.1 \%$ versus $10.5 \%$, respectively; $P<.001$ ); the most feared complication in the surgery group was rupture of the aneurysm $(81.5 \%)$. This is probably related to handling the sac and the surgical dissection. In contrast, the most feared complications of endovascular management were thromboembolic events (33.3\%), followed by vasospasm (19\%). There is not a unanimous consensus regarding the use of antiplatelet treatment in the endovascular group (Figs 1 and 2): Every group has set up their own protocol, probably based on their clinical experience or derived from other specialties. The differences, associated with the use of stents in the acute stage, may explain why thromboembolic events are quite common in patients managed with flow-diverter procedures.

However, in the setting of acute $\mathrm{SAH}$, dual antiplatelet therapy is still controversial; the risk-benefit profile of potential hemorrhagic and thromboembolic complications should be considered carefully in deciding when to initiate such therapy.

Postprocedural complications were also more frequent after surgery $(35.7 \%)$ than after endovascular treatment $(21.1 \%)(P<.001)$; considering these data together, we can state that endovascular treatment is generally associated with fewer complications.

The different treatment groups (endovascular and surgical) were also compared in an attempt to determine whether the type of treatment affects the outcome in some way (Tables 4 and 5).

Considering the numerically larger subgroups, the percentage of good outcomes ( $\mathrm{mRS} 0-2$ ) in the endovascular group was high using flow diverters $(82 \%)$, stent-assisted coiling $(85.2 \%)$, and non-flow-diverter stents $(86.4 \%)$, though the best results were obtained with parent artery occlusion $(88.9 \%)(P=.266)$. Within the group treated surgically, clipping, trapping with bypass, and wrapping with clipping were the more successful techniques $(P=.12)$. These analyses were not statistically significant because of the marked fragmentation of the groups investigated; the other percentages could not be considered reliable because of the small 
sizes of the subgroups. In multivariable analysis, the type of treatment did not seem to influence the outcome (Table 3).

\section{CONCLUSIONS}

Blister-like aneurysms are challenging lesions to treat whether by an open surgery or endovascular techniques. These complex aneurysms often require multiple treatments and have a high incidence of rebleeding. No single approach is clearly superior for these vascular lesions, but several options exist; endovascular treatment seems to be associated with lower morbidity and mortality compared with surgical approaches. The only parameters that seem to influence the outcome are $\mathrm{HH}$ and Fisher grades. Larger and homogeneous cohorts of patients will help to elucidate the optimal treatment for patients with subarachnoid hemorrhage due to blister-like aneurysms.

\section{ACKNOWLEDGMENTS}

The authors thank Enrico Di Stasio, MD, for statistical analysis.

Disclosures: Simone Peschillo—UNRELATED: Consultancy: Educational Proctor for Penumbra.

\section{REFERENCES}

1. Nakagawa F, Kobayashi S, Takemae T, et al. Aneurysms protruding from the dorsal wall of the internal carotid artery. J Neurosurg 1986; 65:303-08 Medline
2. Takahashi A, Suzuki J, Fujiwara S, et al. Surgical treatment of chimame (blood blister-like) aneurysm at c2 portion of internal carotid artery [in Japanese]. Surg Cereb Stroke 1988;16:72-77

3. Ishikawa T, Nakamura N, Houkin K, et al. Pathological consideration of a "blister-like" aneurysm at the superior wall of the internal carotid artery: case report. Neurosurgery 1997;40:403-05; discussion 405-06 Medline

4. Peschillo S, Missori P, Piano M, et al. Blister-like aneurysms of middle cerebral artery: a multicenter retrospective review of diagnosis and treatment in three patients. Neurosurg Rev 2015;38:197-202; discussion 202-03 CrossRef Medline

5. Peschillo S, Cannizzaro D, Missori P, et al. Reconstructive endovascular treatment of a ruptured blood blister-like aneurysm of anterior communicating artery. J Neurosurg Sci 2014 Jun 10. [Epub ahead of print] Medline

6. Andaluz N, Zuccarello M. Blister-like aneurysms of the anterior communicating artery: a retrospective review of diagnosis and treatment in five patients. Neurosurgery 2008;624:807-11; discussion 811 CrossRef Medline

7. Peschillo S, Miscusi, M, Caporlingua A, et al. Blister-like aneurysms in atypical locations: a single-center experience and comprehensive literature review. World Neurosurg 2015;84:1070-79 CrossRef Medline

8. Gonzalez AM, Narata AP, Yilmaz $\mathrm{H}$, et al. Blood blister-like aneurysms: single center experience and systematic literature review. Eur J Radiol 2014;83:197-205 CrossRef Medline

9. Mizutani T, Miki Y, Kojima H, et al. Proposed classification of nonatherosclerotic cerebral fusiform and dissecting aneurysms. Neurosurgery 1999;45:253-59; discussion 259-60 Medline 